\section{Multiple Stercoral Ulcers Caused by Habitual Excessive Daily Intake of Natto (Fermented Soybeans)}
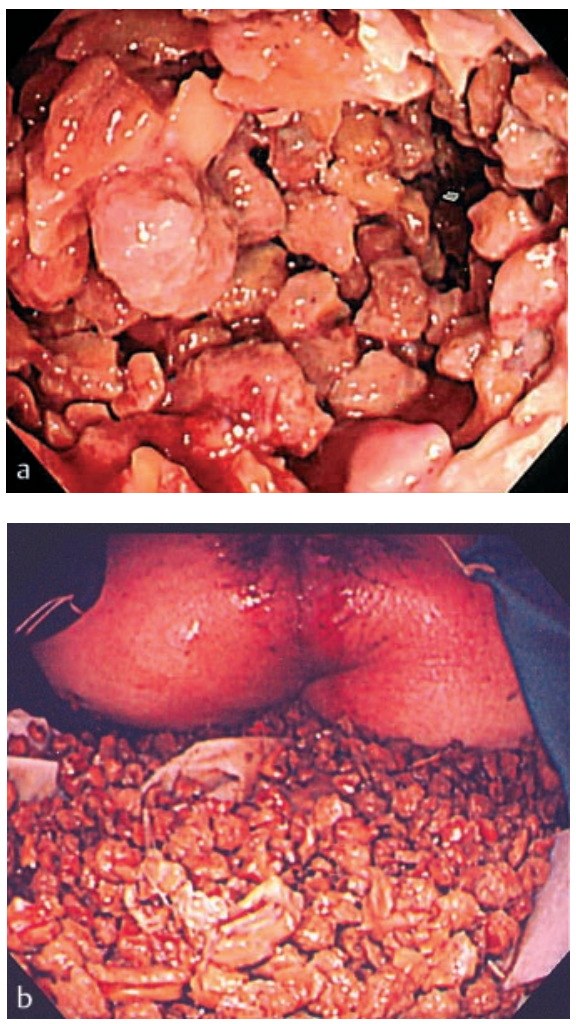

Figure 1 Multiple small fecal stones. a The initial colonoscopic examination showed the stones closely packed in the rectum. b The stones were removed by rectal lavage, performed under saddle-block anesthesia.

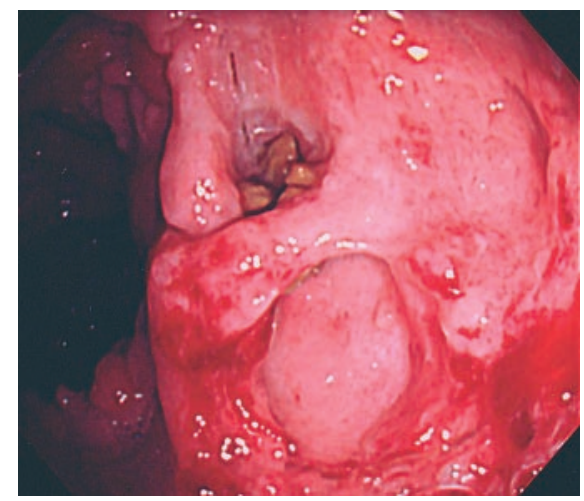

Figure 2 Colonoscopic examination revealed multiple ulcers in the rectum. Incarcerated fecal stones were seen in the largest and deepest ulcer.
It is well known that fecal stones are a rare cause of rectal ulcers: these are known as "stercoral" ulcers [1]. Stercoral ulcers result from pressure necrosis caused by a fecal mass, and they occur most commonly as an isolated lesion in the rectum or sigmoid colon [2]. We encountered a patient with multiple rectal ulcers caused by numerous fecal stones, which were thought to have formed as a result of habitual excessive intake of "natto" (fermented soybeans), a renowned Japanese health food.

A 76-year-old man was admitted to our hospital because of anal bleeding and
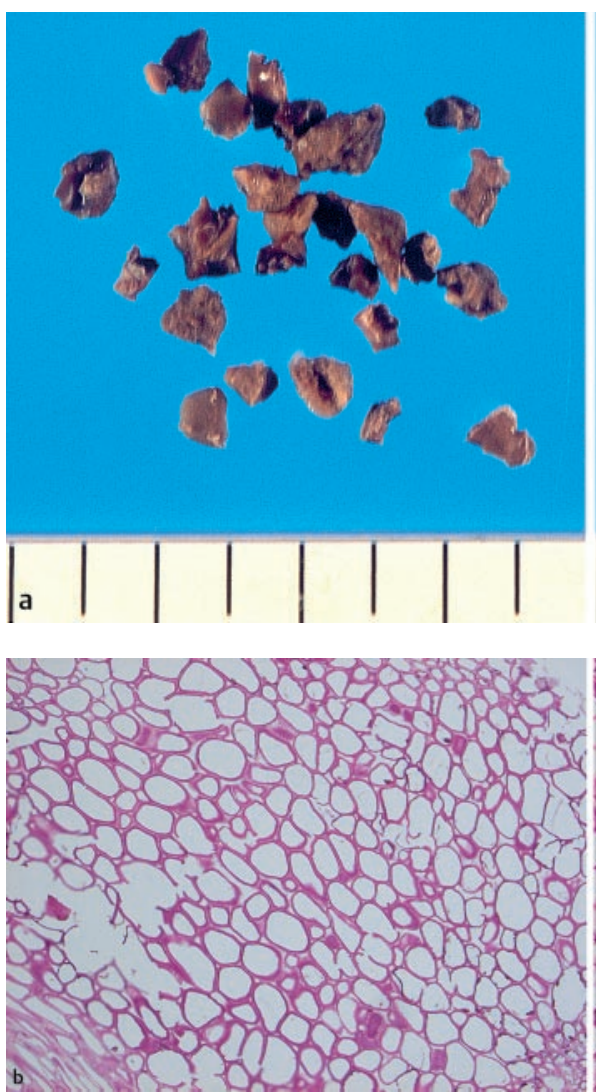

Figure 3 a Macroscopically, the fecal stones (left) were similar in appearance to desiccated natto (right). b Microscopically, the fecal

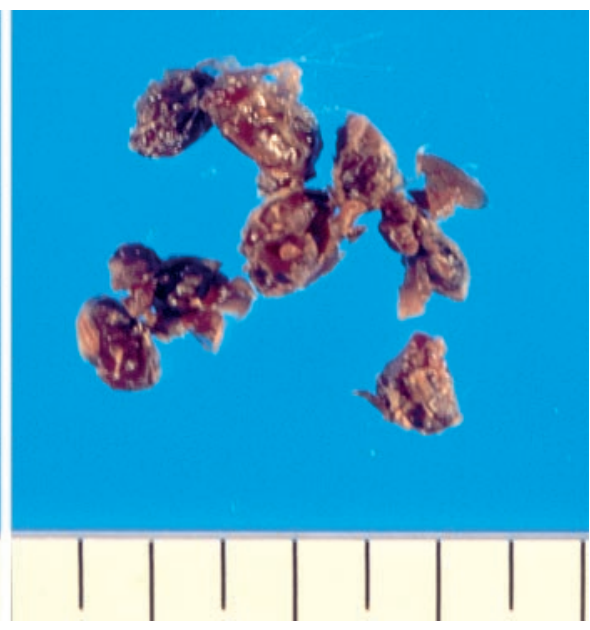

pain. On digital examination we found numerous fecal stones and fresh bleeding. The initial colonoscopic examination showed multiple small fecal stones, which were closely packed in the rectum (Figure 1a). After the stones were removed by rectal lavage under saddleblock anesthesia (Figure $\mathbf{1 b}$ ), colonoscopic examination revealed multiple, round ulcers in the anorectal region (Figure 2). It was later discovered that the patient habitually ate excessive amounts of natto. Macroscopically, the fecal stones were similar in appearance to desiccated natto. Microscopically, the fecal stones were also found to have the same mor-

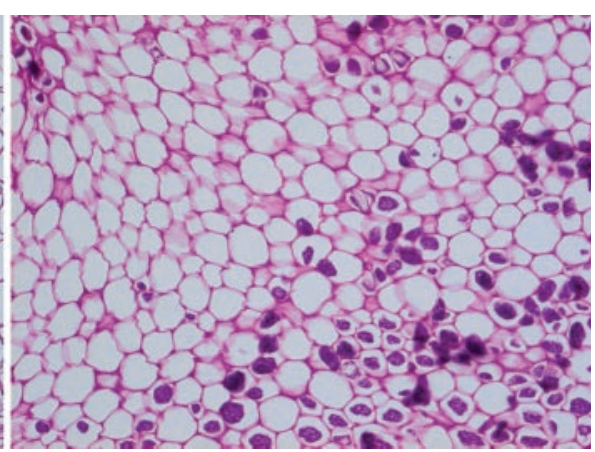

stones (left) and natto (right) showed the same morphological features of the cell walls when stained with periodic acid-Schiff (PAS). 
phological features as natto after staining with periodic acid-Schiff (PAS) stain (Figure $3 \mathbf{a}, \mathbf{b})$. Colonoscopic examination 3 months after initial presentation showed ulcer healing.

This case represents a very rare presentation with multiple rectal ulcers caused by numerous fecal stones, also known as stercoral ulcers. An interesting aspect of this case was that the fecal stones were considered to have been caused by habitual excessive daily intake of natto over a 3-year period. Natto consists of sticky, fermented soybeans, the fermenting agent being the natto bacillus. A popular breakfast item, natto is a foodstuff generally found only in Japan. Recently, however, the number of people who eat this food has been gradually increasing, even in Western countries, because it is regarded as a health food as part of a balanced diet.
S. Kasamaki', T. Kamano², Y. Hayashida ${ }^{3}$, H. Motoyama', H. Yokota ${ }^{1}$

${ }^{1}$ Department of Surgery, Hokota Hospital, Ibaraki Prefecture, Japan

2 Department of Coloproctological Surgery, Juntendo University School of Medicine, Tokyo, Japan

${ }^{3}$ Department of General Medicine, Juntendo University School of Medicine, Tokyo, Japan.

\section{References}

${ }^{1}$ Maull KI, Kinning WK, Kay S. Stercoral ulceration. Am Surg 1982; 48: 20-24

${ }^{2}$ Kingge KL, Katon RM. Massive hematochezia from a visible vessel within a stercoral ulcer: effective endoscopic therapy. Gastrointest Endosc 1997; 46: $369-370$
Corresponding Author

\section{S. Kasamaki, M.D.}

Department of Coloproctological Surgery Juntendo University

School of Medicine

Hongo 2-1-1

Bunkyo-ku

Tokyo 113-8421

Japan

Fax: $\quad$ +81-3-3812-1056

E-mail: kasamake@hh.iij4u.or.jp 